\title{
Silica Chloride Mediated Alkylation of Electron-rich Aromatics by Benzyl or tert-Butyl Chloride
}

\author{
Farajollah Mohanazadeh* and Homira Amini \\ Institute of Chemistry, Iranian Research Organization for Science and Technology, Tehran, Iran \\ *E-mail: mohanazadeh@irost.org \\ Received May 15, 2010, Accepted July 7, 2010
}

Key Words: Silica chloride, Alkylation, Fridel-Crafts

Formation of $\mathrm{C}-\mathrm{C}$ bond is one of the most fundamental and useful reactions in organic chemistry. There are several methods for this purpose. Among the chemical reactions, Fridel-Crafts reaction ${ }^{1}$ is widely used in organic synthesis for alkylation of aromatic rings. Many reagents and methodologies are developed for Fridel-Crafts alkylation reactions. ${ }^{2-7}$

The new environmental legislation pushes for the replacement of all liquid acids by solid acid catalysts which are environmentally more friendly catalysts and which lead to minimal pollution and waste. ${ }^{8}$ Also, in the recent years, the use of reagents and catalysts on solid supports has received significant attention. ${ }^{9}$ Silica gel is one of the extensively used surface material supports for different chemical transformations in organic chemistry. ${ }^{10}$ One such modified silica is silica chloride which has been reported to be an efficient reagent for the synthesis of many organic compounds. ${ }^{11}$ The efficiency of silica chloride, under operationally simple conditions, has prompted us to explore the possibility of this catalyst for the alkylation of aromatic rings.

Silica chloride could be prepared conveniently from inexpensive silica gel and thionyl chloride. ${ }^{12}$ We have discovered that silica chloride acts as catalyst for alkylation of electronrich aromatic compounds by benzyl and tert-butyl chloride (Scheme 1).

The procedure is simple and straightforward. The aromatic compound $(0.01 \mathrm{~mol})$ was added to a suspension of silica chloride $(3 \mathrm{~g})$ and alkyl halide $(0.01 \mathrm{~mol})$. The reaction mixture was stirred at $70{ }^{\circ} \mathrm{C}$. TLC monitored the progress of reaction. After completion of the reaction, the mixture was diluted with ethyl acetate and filtered. The solution was washed with sodium carbonate (5\% in water) and water. The organic layer separated and evaporated. The residue was applied to a silica gel column. Elution with $\mathrm{CCl}_{4}$ gave pure product. We also tried a similar reaction in the presence of silica gel and $\mathrm{HCl}$ (gas) without using silica chloride. The reaction was not successful and the starting materials remained intact.

However, we found that this method was applicable for preparation of alkyl aromatic compounds from reaction of tertbutyl chloride and structurally and electronically diverse benzyl

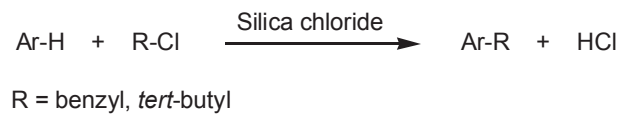

Scheme 1. Reaction of aromatics with benzyl or tert-butyl chloride chloride with aromatic rings substituted with electron-donating groups (Table 1, entries 2-11 and 13-15). The presence of electron-donating groups in aromatics is necessity for progress of

Table 1. Reaction of alkyl halides with aromatic compounds in the presence of silica chloride

Entry Aromatics

${ }^{a}$ The course of reaction was checked by TLC and the ratio of isomers was determined by GC. ${ }^{b}$ Based on aromatic compound. ${ }^{c}$ No reaction. 
reaction, for example, benzene does not react with benzyl chloride (Table 1 , entry 1 ).

Saturated primary and secondary alkyl halides do not react with aromatics in the presence of this reagent and remain mostly intact after the typical reaction times (Table 1, entries 12 and 15).

In conclusion we would like to reiterate that the method is a simple and efficient one, not requiring special equipment or harsh conditions. The respectable yields in these reactions also make it a viable method for the substitution of benzyl or tertbutyl group on aromatic rings.

\section{Experimental Section}

${ }^{1}$ H-NMR spectra were recorded on a Jeol JNM-PMX $60_{\text {SI }}$ NMR spectrometer using tetramethylsilane as the internal standard. IR spectra were taken on a Perkin-Elmer 267 spectrophotometer. Thin layer chromatography was performed on silica gel (Macerey-Nagel Co., plygram Sil G/uv). GC analyses were performed on Philips PU 4500.

All organic solutions were dried over $\mathrm{MgSO}_{4}$ or $\mathrm{Na}_{2} \mathrm{SO}_{4}$ and solvents were removed under reduced pressure on a Buchi evaporator. All alkylation products are known compounds, they were identified by comparison of their spectral (IR, NMR) and physical data with those of authentic samples.

Preparation of silica chloride. Into a flask equipped with a reflux condenser and calcium chloride tube, silica gel (12 g) and thionyl chloride $(100 \mathrm{~mL})$ were charged. The reaction mixture was refluxed under stirring with a magnetic stirrer for $18 \mathrm{~h}$, and the untreated thionyl chloride was removed by distillation. The silica chloride was dried in vacuum at $90{ }^{\circ} \mathrm{C}$ and the resulting grayish powder was stored in a desiccator under vacuum. The chlorosilyl groups on silica surface $(0.9 \mathrm{mmol}$ of $\mathrm{Cl} / \mathrm{g}$ silica $)$ were determined by suspension or reagent $(1 \mathrm{~g})$ in $\mathrm{NaHCO}_{3}$ solution $(0.005 \mathrm{M}, 100 \mathrm{~mL})$. The resulting chloride ion was titrated with silver nitrate solution $(0.01 \mathrm{M}, 90 \mathrm{~mL})$ using potassium chromate solution as indicator.

Reaction of toluene with benzyl chloride, typical procedure. Toluene $(0.92 \mathrm{~g}, 0.01 \mathrm{~mol})$ was added to a suspension of silica chloride (3 g) and benzyl chloride (1.26 g, $0.01 \mathrm{~mol})$. The reaction mixture was stirred at $70{ }^{\circ} \mathrm{C}$, under dried atmosphere, for $8 \mathrm{~h}$. TLC monitored the progress of reaction. After completion of the reaction, the mixture was diluted with ethyl acetate $(25 \mathrm{~mL})$ and filtered. The solution was washed with sodium carbonate (5\% in water) and water. The organic layer separated and evaporated. The residue was applied to a silica gel column. Elution with $\mathrm{CCl}_{4}$ gave mixture of isomers $(1.24 \mathrm{~g}, 68 \%)$. The ratio of isomers was determined by GC (tubular column, $150 \mathrm{ft}$, coated with $m$-bis ( $m$-phenoxyphenoxy)benzene modified with $20 \%$ Apiezon). ${ }^{13-14}$ A hydrogen flame-ionization detector with helium carrier gas of 30 psi was used, columns being operated at $125^{\circ} \mathrm{C}$, with detector temperature of $185-190{ }^{\circ} \mathrm{C}$ and injector block temperature of $310-320^{\circ} \mathrm{C}$. Retention times of 2-methyl-, and 4-methyldiphenylmethane were respectively $111.1 \mathrm{~min}$ and $118.9 \mathrm{~min}$

4-Methyldiphenylmethane: ${ }^{1} \mathrm{H}$ NMR $\left(60 \mathrm{MHz}, \mathrm{CDCl}_{3}\right) \delta$ 7.0-7.2 (m, 9H, ArH), 3.8 (s, 2H, $\left.\mathrm{CH}_{2}\right), 2.3$ (s, 3H, $\left.\mathrm{CH}_{3}\right)$.

2-Methyldiphenylmethane: ${ }^{1} \mathrm{H}$ NMR $\left(60 \mathrm{MHz}, \mathrm{CDCl}_{3}\right) \delta$ 6.9-7.3 (m, 9H, ArH), 3.7 s, 2H, $\mathrm{CH}_{2}$ ), 2.4 (s, 3H, $\mathrm{CH}_{3}$ ).
4-Hydroxydiphenylmethane: ${ }^{1} \mathrm{H}$ NMR $\left(60 \mathrm{MHz}, \mathrm{CDCl}_{3}\right) \delta$ 3.9 (s, 2H, $\mathrm{CH}_{2}$ ), 4.9 (br. s, 1H, OH), 6.7-7.1 (dd, 4H, ArH), 7.2 (s, 5H, ArH). mp $85-87^{\circ}$ (lit. $^{15} 86^{\circ}$ )

2-Hydroxydiphenylmethane: ${ }^{1} \mathrm{H} \mathrm{NMR}\left(60 \mathrm{MHz}, \mathrm{CDCl}_{3}\right) \delta$ 4.0 (br. s, $2 \mathrm{H}, \mathrm{CH}_{2}$ ), 4.9 (s, 1H, OH), 6.6-7.2 (m, 9H, ArH). mp $48.5-51^{\circ}$ (lit. $^{15}$ ).

2-Benzylthiophene: ${ }^{1} \mathrm{H} \mathrm{NMR}\left(60 \mathrm{MHz}, \mathrm{CDCl}_{3}\right) \delta 7.2(\mathrm{~s}, 5 \mathrm{H}$, ArH), 7.0-7.1 (m, 1H, ArH), 6.7-6.9 (m, 2H, ArH), 4.1 (s, 2H, $\mathrm{CH}_{2}$ ). Yellow oil.

4-Methoxydiphenylmethane: ${ }^{1} \mathrm{H} \mathrm{NMR}\left(60 \mathrm{MHz}, \mathrm{CDCl}_{3}\right) \delta$ 6.8-7.8 (m, 9H, ArH), 4.0 (s, 3H, $\left.\mathrm{CH}_{3}\right), 3.8$ (s, 2H, $\left.\mathrm{CH}_{2}\right) . \mathrm{mp}$ $20-21^{\circ}$ (lit. $\left.^{16} 20^{\circ}\right)$

2-Methoxydiphenylmethane: ${ }^{1} \mathrm{H} \mathrm{NMR}\left(60 \mathrm{MHz}, \mathrm{CDCl}_{3}\right) \delta$ 6.6-7.8 (m, 9H, $\mathrm{ArH}), 4.0$ (s, 3H, $\left.\mathrm{CH}_{3}\right), 3.9$ (s, 2H, $\left.\mathrm{CH}_{2}\right)$. mp $38-40^{\circ}$ (lit. $\left.^{16} 39^{\circ}\right)$.

4-Chloro-4'-methyldiphenylmethane: ${ }^{1} \mathrm{H}$ NMR $(60 \mathrm{MHz}$, $\left.\mathrm{CDCl}_{3}\right) \delta$ 7.0-7.3 (m, 8H, ArH), $3.9\left(\mathrm{~s}, 2 \mathrm{H}, \mathrm{CH}_{2}\right), 2.3$ (s, 3H, $\mathrm{CH}_{3}$ ). Colorless oil (lit. ${ }^{17}$ )

4-Chloro-2'-methyldiphenylmethane: ${ }^{1} \mathrm{H}$ NMR $(60 \mathrm{MHz}$, $\left.\mathrm{CDCl}_{3}\right) \delta$ 7.0-7.5 (m, $\left.8 \mathrm{H}, \mathrm{ArH}\right), 3.8\left(\mathrm{~s}, 2 \mathrm{H}, \mathrm{CH}_{2}\right), 2.4(\mathrm{~s}, 3 \mathrm{H}$, $\mathrm{CH}_{3}$ ). Colorless oil (lit. ${ }^{17}$ ).

4-Methoxy-4'-methyldiphenylmethane: ${ }^{1} \mathrm{H} \mathrm{NMR}(60 \mathrm{MHz}$, $\left.\mathrm{CDCl}_{3}\right) \delta$ 6.9-7.4 (m, 8H, ArH), $4.0\left(\mathrm{~s}, 3 \mathrm{H}, \mathrm{CH}_{3}\right), 3.8(\mathrm{~s}, 2 \mathrm{H}$, $\left.\mathrm{CH}_{2}\right), 2.3$ (s, $\left.3 \mathrm{H}, \mathrm{CH}_{3}\right)$. Colorless oil (lit. ${ }^{18}$ ).

4-Methoxy-2'-methyldiphenylmethane: ${ }^{1} \mathrm{H} \mathrm{NMR}(60 \mathrm{MHz}$, $\left.\mathrm{CDCl}_{3}\right) \delta$ 6.8-7.7 (m, 8H, ArH), $4.0\left(\mathrm{~s}, 3 \mathrm{H}, \mathrm{CH}_{3}\right), 3.9(\mathrm{~s}, 2 \mathrm{H}$, $\mathrm{CH}_{2}$ ), 2.4 (s, 2H, $\mathrm{CH}_{2}$ ). Colorless oil (lit. ${ }^{18}$ ).

4-Nitro-4'-methyldiphenylmethane: ${ }^{1} \mathrm{H}$ NMR $(60 \mathrm{MHz}$, $\left.\mathrm{CDCl}_{3}\right) \delta 7.1-7.5(\mathrm{~m}, 8 \mathrm{H}, \mathrm{ArH}), 4.0\left(\mathrm{~s}, 2 \mathrm{H}, \mathrm{CH}_{2}\right), 2.5(\mathrm{~s}, 3 \mathrm{H}$, $\mathrm{CH}_{3}$ ). $\mathrm{bp}_{12}$ 195-6-8 (lit. ${ }^{19} \mathrm{bp}_{12}$ 195-8 ${ }^{\circ}$ ).

4-Nitro-2'-methyldiphenylmethane: ${ }^{1} \mathrm{H}$ NMR $(60 \mathrm{MHz}$, $\left.\mathrm{CDCl}_{3}\right) \delta$ 7.0-7.6 (m, 8H, ArH), $4.1\left(\mathrm{~s}, 2 \mathrm{H}, \mathrm{CH}_{2}\right), 2.5(\mathrm{~s}, 3 \mathrm{H}$, $\mathrm{CH}_{3}$ ). Oil (lit. ${ }^{19}$ ).

4-tert-Butyltoluene: ${ }^{1} \mathrm{H}$ NMR $\left(60 \mathrm{MHz}, \mathrm{CDCl}_{3}\right) \delta$ 7.0-7.3 (m, $4 \mathrm{H}, \mathrm{ArH}), 2.3\left(\mathrm{~s}, 3 \mathrm{H}, \mathrm{CH}_{3}\right), 1.3\left(\mathrm{~s}, 9 \mathrm{H}, 3 \mathrm{CH}_{3}\right)$. bp $189-194^{\circ}$ (lit. $^{20} 189-92^{\circ}$ ).

2-tert-Butyltoluene: ${ }^{1} \mathrm{H}$ NMR $\left(60 \mathrm{MHz}, \mathrm{CDCl}_{3}\right) \delta$ 6.9-7.5 (m, 4H, ArH), 2.4 (s, 3H, $\left.\mathrm{CH}_{3}\right), 1.3\left(\mathrm{~s}, 9 \mathrm{H}, 3 \mathrm{CH}_{3}\right)$. bp $\mathrm{p}_{743} 169$ $72^{\circ}$ (lit. $\left.^{20} 170-71^{\circ}\right)$.

4-tert-Butylphenol: ${ }^{1} \mathrm{H}$ NMR $\left(60 \mathrm{MHz}, \mathrm{CDCl}_{3}\right) \delta$ 6.4-7.3 (dd, 4H, ArH), 5.0 (br. s, 1H, OH), 1,3 (s, 9H, 3CH $)$. mp 98 $9{ }^{\circ} \mathrm{C}\left(\right.$ lit. $\left.^{21} 99^{\circ}\right)$.

2-tert-Butylphenol: ${ }^{1} \mathrm{H}$ NMR $\left(60 \mathrm{MHz}, \mathrm{CDCl}_{3}\right) \delta$ 6.4-7.5 (m, 4H, ArH), 4.8 (br. s, 1H, OH), 1.4 (s, 9H, 3CH $)$. bp 222 $4^{\circ}$ (lit. $^{22} 224^{\circ}$ )

4-tert-Butylanisole: ${ }^{1} \mathrm{H}$ NMR $\left(60 \mathrm{MHz}, \mathrm{CDCl}_{3}\right) \delta$ 6.9-7.3 (dd, $4 \mathrm{H}, \mathrm{ArH}), 3.9$ (s, 3H, $\left.\mathrm{CH}_{3}\right), 1.3\left(\mathrm{~s}, 9 \mathrm{H}, 3 \mathrm{CH}_{3}\right)$. bp $214-6^{\circ}$ (lit. $\left.{ }^{21} 215-6\right)$.

2-tert-Butylanisole: ${ }^{1} \mathrm{H} \mathrm{NMR}\left(60 \mathrm{MHz}, \mathrm{CDCl}_{3}\right) \delta$ 6.8-7.5 (m, $4 \mathrm{H}, \mathrm{ArH}), 3.9\left(\mathrm{~s}, 3 \mathrm{H}, \mathrm{CH}_{3}\right), 1.4\left(\mathrm{~s}, 9 \mathrm{H}, 3 \mathrm{CH}_{3}\right) . \mathrm{bp}_{22} 102-106^{\circ}$ (lit. $\left.{ }^{22} \mathrm{bp}_{22} 102.5-104.5^{\circ}\right)$.

2-tert-Butylthiophene: ${ }^{1} \mathrm{H} \mathrm{NMR}\left(60 \mathrm{MHz}, \mathrm{CDCl}_{3}\right) \delta$ 6.7-6.9 (m, 2H, ArH), 7.1-7.2 (m, 1H, ArH), $1.1\left(\mathrm{~s}, 9 \mathrm{H}, 3 \mathrm{CH}_{3}\right) . \mathrm{bp}_{0.2}$ $74-7^{\circ}$ (lit. $^{23} \mathrm{bp}_{0.15} 75^{\circ}$ ).

2-tert-Butylfurane: ${ }^{1} \mathrm{H}$ NMR $\left(60 \mathrm{MHz}, \mathrm{CDCl}_{3}\right) \delta 7.3(\mathrm{~s}, 1 \mathrm{H}$, ArH), 6.2-6.3 (m, 1H, ArH), 5.9-6.1 (m, 1H, ArH). bp $114-6^{\circ}$ (lit. $\left.^{23} 115.5^{\circ}\right)$.

2-(2-Chloro-1-phenylethyl)thiophene: ${ }^{1} \mathrm{H}$ NMR (60 MHz, 
$\left.\mathrm{CDCl}_{3}\right) \delta$ 7.1-7.3 (br.s, 5H, Arh), 7.1-7.2 (m, 1H, ArH), 6.66.6.9 (m, 2H, ArH). Colorless liquid.

\section{References}

1. Olah, G. A. Fridel-Crafts Chemistry; Wiley and Son: New York, 1973.

2. Olah, G. A.; Kobayashi, S.; Tashiro, M. J. Am. Chem. Soc. 1972 , 94, 7448 .

3. Khalaf, A. A.; Roberts, R. M. J. Org. Chem. 1970, 35, 3717.

4. Khalaf, A. A.; Raberts, R. M. J. Org. Chem. 1972, 37, 4227.

5. Wang, X.; Wang, Y.; Du, D. M.; Xu, J. Journal of Molecular Catalysis A: Chemical 2006, 255, 31.

6. Schaarschmidt, A.; Hermann, L.; Szemzo, B. Ber. 1925, 58B, 1914

7. Roberts, R. M.; El-Khawaga, A. M.; Roengsumran, S. J. Org. Chem. 1984, 49, 3180.

8. Clark, J. H.; Cullen, S. R.; Barlow, S. J.; Bastock, T. W. J. Chem. Soc. Perkin Trans 2 1994, 1117.

9. (a) McKillop, P. A.; Young, D. W. Synthesis 1979, 401. (b) Cornelis, A.; Laszlo, P. Synthesis 1985, 909. (c) Zhu, J. Eur. J. Org. Chem. 2003, 2003, 1133.

10. (a) Tal, D. M.; Keinan, E.; Mazur, Y. Tetrahedron 1981, 37, 4327.

(b) Onofrio, F. D.; Scettri, A. Synthesis 1985, 1159

11. (a) Kamitori, Y.; Hojo, M.; Masuda, R.; Kimura, T.; Yoshida, T.
J. Org. Chem. 1986, 51, 1427. (b) Ravindranath, N.; Ramesh, C.; Das, B. Synlett 2001, 1777. (c) Sathe, M.; Kaushik, M. P. Catalysis Communications 2006, 644. (d) Sathe, M.; Gupta, A. K.; Kaushik, M. P. Tetrahedron Lett. 2006, 47, 3107. (e) Mohanazadeh, F.; Momeni, A. R.; Ranjbar, Y. Tetrahedron Lett. 1994, 35, 6127.

12. Saunders, D. H.; Barford, R. A.; Magidman, P.; Olszewski, L. T.; Rothbart, H. L. Anal. Chem. 1974, 46, 834.

13. Walker, J. Q. Anal. Chem. 1963, 35, 2028.

14. Olah, G. A.; Olah, J. A. J. Org. Chem. 1967, 32, 1612.

15. Tarbell, D. S.; Petropoulos, J. C. J. Am. Chem. Soc. 1952, 74, 244.

16. Papa, D.; Schwenk, E.; Whitman, B. J. Org. Chem. 1942, 7, 587.

17. Darbeau, R. W.; White, E. H.; Song, F.; Darbeau, N. R.; Chou, J. J. Org. Chem. 1999, 64, 5966.

18. Lipshutz, B. H.; Tomioka, T.; Blomgren, P. A.; Sclafani, J. A. Inorganica Chimica Acta 1999, 269, 164

19. Kliegl, A. Ber. 1908, 41, 1847.

20. Schlatter, M. J.; Clark, R. D. J. Am. Chem. Soc. 1953, 75, 361.

21. Bowman, R. S.; Stevens, D. R.; Baldwin, W. E. J. Am. Chem. Soc. $1957,79,87$.

22. Goldsmith, E. A.; Schlatter, M. J.; Toland, W. G. J. Org. Chem. 1958, 23, 1871.

23. Cadogan, J. I. G.; Hey, D. H.; Sanderson, W. A. J. Chem. Soc. 1960, 3203.

24. Shuikin, N. I.; Lebedev, B. L.; Nikolskil, V. G.; Korytina, O. A.; Kessenikh, A. V.; Prokofev, E. P. Russ. Chem. Bull. 1967, 16, 1561. 